\title{
Ohne Erfolgsgarantie
}

\section{Psychotherapie ist kein Wundermittel bei chronischen Schmerzen}

\author{
Für chronische Schmerzpatienten gibt es kein Wunderheilmittel, warnen Schmerzexperten. Bei \\ somatoformen Schmerzstörungen sei eine frühzeitige und intensive Psychotherapie essenziell. Dabei \\ geht es aber eher darum, anders mit den Schmerzen leben zu lernen, als um reine Schmerzreduktion.
}

\begin{abstract}
ei chronischen Schmerzpatienten
kann man von keiner Therapie erwarten, dass sie garantiert zur Schmerzfreiheit führt. Im Rahmen einer Psychotherapie geht es eher darum, das Leiden am Schmerz zu verringern sowie soziale und alltagspraktische Fähigkeiten zu fördern. Wer unter einer solchen Behandlung dann doch eine mehr als $50 \%$ ige Schmerzreduktion erfährt, hat größere Chancen, dass dieser Effekt auch nach Beendigung der Behandlung noch weiter anhält als bei der Pharmakotherapie. PD Dr. Winfried Häuser, Leiter des Schwerpunkts Psychosomatik, Innere Medizin I, Klinikum Saarbrücken, warnt jedoch vor überzogenen Erwartungen. Bei vielen Patienten mit somatoformen Schmerzstörungen sei der chronische Schmerz nur die Spitze des Eisbergs multipler psychischer und körperlicher Probleme. Häuser hält dann eine frühzeitige und ausreichend intensive Behandlung für essenziell.
\end{abstract}

\section{„Palliative psychosomatische Grundversorgung"}

Psychosomatische Schmerzexperten stellten vor kurzem in einer Übersichtsarbeit eine „Unterdosierung von Psychotherapie“ im Rahmen vieler multimodaler Verfahren fest [1]. Ähnlich wie bei einer fortgeschrittenen Krebserkrankung, die auf mehrere Chemotherapiekombinationen nicht angesprochen hat, solle man aber, so Häuser, bei wiederholt psychotherapieresistenten Schmerzerkrankungen auch einen palliativen Ansatz und den „Verzicht auf die vierte Schmerzpsychotherapie oder fünfte stationäre multimodale Schmerztherapie “ in Erwägung ziehen. Eine solche „palliative psychosomatische Grundversorgung" nach Häu- sers Vorstellung umfasst niedrigfrequente, nicht-beschwerdekongruente Kontakte und die Möglichkeit zur Krisenintervention. Gleichzeitig werden nicht-indizierte Therapien wie hoch dosierte Opioide oder invasive Verfahren und Operationen vermieden. Ein realistisches Ziel ist dabei, das derzeitige soziale und alltagspraktische Funktionsniveau des Patienten zu erhalten oder zumindest deren Verschlechterung zu verlangsamen.

Thomas Bißwanger-Heim

Symposium „Psychotherapie von Schmerzpatienten - Wie geht das? Praktische Beispiele aus verschiedenen Bereichen und Schulen - Gemeinsames und Verschiedenes", 18.10.2012, Deutscher Schmerzkongress 2012, Mannheim

\section{Kopfschmerz}

\section{Kennen Sie Airplane headache?}

\author{
In der Klassifikation der internationalen Kopfschmerzgesellschaft (IHS) \\ sind inzwischen mehr als 255 verschiedene Kopfschmerztypen aufge- \\ listet. Airplane headache ist noch nicht dabei.
}

E rstmals im Jahre 2004 beobachtet und bislang noch nicht in der IHS-Klassifikation vertreten ist der Kopfschmerz im Verlauf von Flugreisen. Der Airplane headache zählt zu den Kopfschmerzformen der Gruppe 10 der IHS, die durch Störungen der Homöostase charakterisiert sind, so PD Dr. Stefanie Förderreuther, München. Die akuten, stets stereotyp verlaufenden stechenden und streng einseitigen Schmerzen treten typischerweise sehr rasch im Landeanflug auf. Sie klingen meist binnen 15-30 Minuten $\mathrm{ab}$, nur vereinzelt finden sich prolongierte Verläufe. Jeweils ein Viertel der Patienten berichte über regelmäßige Attacken dieser Art beziehungsweise über Augensymptome wie Rötung, Myosis oder Tränenfluss. Migränetypische Symptome wie Photo- oder Phonophobie fehlen dagegen völlig.
Airplane headache tritt deutlich häufiger bei Männern auf und in der Anamnese finden sich keine anderen Kopfschmerztypen. „Sie sollten diesen seltenen Kopfschmerz kennen, weil die Patienten davon Angst bekommen“, so Förderreuther. Ausgeschlossen werden sollte eine Beteiligung der Nasennebenhöhlen, die ebenfalls im Landeanflug vermehrt Beschwerden machen kann. Hilfreich gegen Airplane headache kann bei bekannter Empfindlichkeit die Prophylaxe mit Naproxen sein.

Dr. Andreas Häckel

Update Kopfschmerz: Das Wichtigste der letzten 2 Jahre zu Migräne, trigemino-autonomen Kopfschmerzen, Kopfschmerzen zurückzuführen auf eine Somatisierungsstörung und zu Kopfschmerzen als Folge einer Störung der Homöostase, Deutscher Schmerzkongress 2012, Mannheim 